\title{
Evolução e variabilidade espacial da salinidade em Neossolo Flúvico cultivado com cenoura sob irrigação ${ }^{1}$
}

\author{
Edivan R. de Souza ${ }^{2}$, Abelardo A. A. Montenegro ${ }^{2}$ \& Maria B. G. dos S. Freire $^{2}$
}

\begin{abstract}
RESUMO
Desenvolveu-se um experimento de campo com o intuito de verificar o efeito da irrigação por microaspersão, utilizando-se água de condutividade elétrica média de $1,42 \mathrm{dS} \mathrm{m}^{-1}$, sob as propriedades químicas de um Neossolo Flúvico cultivado com cenoura cv. Brasília. A área foi dividida em dois setores $\left(S_{1}\right.$ e $\left.S_{2}\right)$, com $900 \mathrm{~m}^{2}$ cada um $(30$ x $30 \mathrm{~m})$ e texturas contrastantes; no $S_{2}$ adicionou-se cobertura morta aos 23 dias após a semeadura (DAS) e se adotou uma fração de lixiviação de 0,20 para os dois setores. Realizaram-se duas amostragens de solo, antes da semeadura e a 96 DAS, nas camadas de 0-20 e 20-40 cm, em 49 pontos no $S_{1}$ e 52 pontos no $S_{2}$, segundo uma malha de $5 \times 5 \mathrm{~m}$. No extrato da pasta saturada foram medidos o $\mathrm{pH}$ e a condutividade elétrica $\left(\mathrm{CE}_{\mathrm{es}}\right)$, e determinadas as bases solúveis; determinou-se, ainda, as bases trocáveis, calculando-se a relação de adsorção de sódio e a percentagem de sódio trocável. Os dados foram analisados por estatística descritiva e geoestatística. Considerando-se a camada de $0-40 \mathrm{~cm}$, a área salina no $\mathrm{S}_{1}$, inicialmente de 7,98\%, aumentou para 15,09\% ao final do ciclo cultural. Para o $S_{2}$, a área salina passou de 5,97 para $5,52 \%$; verificou-se, assim, a influência decisiva da textura e da cobertura morta no controle da salinidade do solo.
\end{abstract}

Palavras-chave: geoestatística, relação de adsorção de sódio, percentagem de sódio trocável, Daucus carota L.

\section{Evolution and spatial variability of salinity in a Fluvic Neossol cropped with irrigated carrot}

\begin{abstract}
A field experiment was carried out aiming to verify the effect of micro sprinkler irrigation, using water with an average electrical conductivity of $1.42 \mathrm{dS} \mathrm{m}^{-1}$, on the chemical properties of a Fluvic Neossol cropped with carrot, variety Brasília. The area was divided into two sectors $\left(S_{1}\right.$ and $\left.S_{2}\right)$, each one with $900 \mathrm{~m}^{2}(30 \times 30 \mathrm{~m})$ and contrasting textures; at $S_{2}$ mulch was added at day 23 after sowing (DAS). A leaching fraction of 0.20 was adopted for both sectors. Two soil sampling schemes were carried out, before sowing and at 96 DAS, in the 0-20 and 20-40 cm layers, at 49 points in $S_{1}$ and 52 points in $S_{2}$, according to a regular $5 \times 5 \mathrm{~m}$ mesh. In the extract of saturated soil paste, $\mathrm{pH}$ and electrical conductivity $\left(\mathrm{EC}_{\mathrm{se}}\right)$ were measured, and exchangeable cations in soil were evaluated; in addition, the sodium adsorption ratio and the exchangeable sodium percentage were calculated. Data has been analysed using descriptive statistics and geostatistics. Considering the $0-40 \mathrm{~cm}$ layer, the saline area at $S_{1}$, covering $7.98 \%$ at the beginning, increased to $15.09 \%$ by the end of the cropping period. For $S_{2}$, the percentage of saline area shifted from $5.97 \%$ to $5.52 \%$. The decisive influence of texture and mulch for the control of soil salinity has thus been verified.
\end{abstract}

Key words: geoestatistics, sodium adsorption ratio, exchangeable sodium percentage, Daucus carota L.

${ }^{1}$ Extraído da Dissertação do primeiro autor apresentada ao Programa de Pós-Graduação em Ciência do Solo/UFRPE

2 UFRPE. Rua Dom Manoel de Medeiros s/n - Dois Irmãos, CEP 52171-900. Recife, PE. Fone: (81) 3320-6260. E-mails: edivanrs@hotmail.com; monte@hotlink.com.br; betania@depa.ufrpe.br 


\section{INTRODUÇÃO}

A salinização do solo é um dos problemas mais sérios para a agricultura irrigada, sobretudo em regiões semi-áridas e áridas, e pode ser causada pelo manejo inadequado da irrigação, especialmente quando se utiliza água salobra (Horney et al., 2005). Ribeiro et al. (2003) comentam que nessas regiões, em que as condições climáticas favorecem o enriquecimento químico dos recursos hídricos, as águas contêm sais dissolvidos passíveis de comprometer a qualidade do solo a médio e longo prazo. O excesso de sais no solo pode prejudicar o crescimento das plantas em virtude dos efeitos osmóticos e da toxicidade de íons específicos presentes em elevadas concentrações na solução do solo (Freire et al., 2003a).

Audry \& Suassuna (1995) encontraram, avaliando a qualidade das águas de riachos, açudes e poços do semi-árido do Nordeste, a maior incidência de água com problemas de salinidade e sodicidade nas águas provenientes de poços tubulares, o que exige um manejo diferenciado para essas águas. Dias et al. (2004), estudando a evolução da salinidade em um Argissolo cultivado com melão, utilizaram águas de irrigação com três níveis salinos: 1,2, 2,5 e 4,5 dS m¹ e concluíram que, aos 45 dias após o transplantio, a salinidade do solo apresentou um acréscimo atribuído, em parte, à aplicação de fertilizantes via fertirrigação e à reduzida fração de lixiviação, que foi de 0,10 .

O acúmulo de sais no solo depende também das suas propriedades físicas e, sobretudo, das condições de drenagem e do balanço de água e de sais na zona radicular (Dias et al., 2005). Desse modo, dependendo da salinidade da água e do solo, é recomendável a adoção de uma fração de lixiviação, associada a um sistema de drenagem adequado.

Ainda há carência de estudos sobre salinidade abordando diferentes tipos de solo e culturas, verificando não só o comportamento dos solos, mas também a tolerância das culturas exploradas em regiões semi-áridas. Ayers \& Westcot (1991) classificam a cenoura como sensível à salinidade, com uma condutividade elétrica do extrato saturado $\left(\mathrm{CE}_{\mathrm{es}}\right)$ limitante de $1,0 \mathrm{dS} \mathrm{m}^{-1}$ e condutividade elétrica da água de irrigação $\left(\mathrm{CE}_{\mathrm{a}}\right)$ de $0,70 \mathrm{dS} \mathrm{m}^{-1}$. Valores acima dos mencionados tendem a provocar redução do rendimento potencial da cultura; por exemplo, $\mathrm{CE}_{\mathrm{es}}$ de $2,8 \mathrm{dS} \mathrm{m}^{-1}$ e CEa de $1,9 \mathrm{dS} \mathrm{m}^{-1}$, haveria perdas de $25 \%$ na produtividade relativa, segundo esses autores.

Outro aspecto importante quando se trabalha com salinização em áreas irrigadas, é a abordagem da variabilidade espacial do teor de sais, resultante das lâminas aplicadas e da heterogeneidade das propriedades físicas. Diversas metodologias são adotadas podendo-se trabalhar com métodos estatísticos, através do quais são formulados tratamentos dispostos em blocos homogêneos. Alternativamente, a geoestatística é outra maneira de se avaliar os resultados, requerendo que sejam conhecidas as distâncias de separação entre os pontos monitorados, para identificar a distância de dependência espacial da variável estudada (Vieira et al., 2002). Com os resultados dos parâmetros do semivariograma (efeito pepita, patamar e alcance) é possível, através da krigagem, o mapeamento da propriedade de interesse considerando-se a correlação existente.
Desta forma, se objetivou, com este trabalho, estudar a evolução da salinidade e sodicidade de um Neossolo Flúvico cultivado com cenoura irrigada com água moderadamente salina. Buscou-se avaliar a variabilidade espacial dessas variáveis, identificando-se as áreas afetadas por sais, e o desempenho de alternativa de manejo com lixiviação incompleta e cobertura morta na produtividade da cenoura.

\section{MATERIAL E MÉTODOS}

A área do experimento está localizada na sub-bacia do Rio Ipanema, no Estado de Pernambuco, entre as coordenadas $08^{\circ} 34^{\prime} 17^{\prime}$ de Latitude Sul e $37^{\circ} 01^{\prime} 20^{\prime}$ ' de Longitude Oeste, com altitude média de $613 \mathrm{~m}$, no município de Pesqueira e com solo caracterizado como Neossolo Flúvico (Ribeiro \& Corrêa, 2001).

A região apresenta precipitação total anual média de 730 mm e evapotranspiração potencial de 1.683 mm. A área de trabalho vem sendo explorada por agricultores familiares aproximadamente há dezesseis anos, principalmente com o cultivo de hortaliças, dentre elas pimentão, repolho e cenoura.

O experimento foi conduzido no período de 29 de outubro de 2005 a 02 de fevereiro de 2006. O preparo da área consistiu de aração e gradagem e uma adubação de fundação nas quantidades $40 \mathrm{~kg} \mathrm{ha}^{-1}$ de $\mathrm{N} ; 60 \mathrm{~kg} \mathrm{ha}^{-1}$ de $\mathrm{K}_{2} \mathrm{O}$ e $60 \mathrm{~kg} \mathrm{ha}^{-1}$ de $\mathrm{P}_{2} \mathrm{O}_{5}$ nas formas de uréia, cloreto de potássio e fosfato mono amônio, com base na análise de solo e nas necessidades da cultura, conforme as recomendações para a cultura da cenoura no Estado de Pernambuco (CEFS, 1998). Após o preparo da área se confeccionaram os canteiros para o plantio da cenoura $(30 \times 1,0 \times 0,20 \mathrm{~m})$ e se utilizou a variedade Brasília por ser recomendada para as condições do Nordeste brasileiro e adotada pelos agricultores da região estudada.

O sistema de irrigação adotado foi o localizado-microaspersão, com emissores espaçados 4 x $3 \mathrm{~m}$, com arranjo triangular. Instalaram-se dois setores de irrigação, cada um com área de $900 \mathrm{~m}^{2}$ (30 x $30 \mathrm{~m}$ ), denominados setores $\mathrm{S}_{1}$ e $\mathrm{S}_{2}$. Para monitoramento da umidade do solo ao longo do ciclo da cultura, foram instalados tubos de acesso de PVC para sonda de nêutrons. No $S_{1}$ se instalaram 49 tubos, enquanto no $S_{2}$ esse número foi de 52 tubos de acesso. Os locais de instalação dos tubos coincidiram com os nós de uma malha de $5 \times 5 \mathrm{~m}$, onde coletaram-se amostras de solo para análises físicas e químicas em todos os nós, nas camadas de 0-20 e 20-40 cm, antes da semeadura e sempre ao redor dos tubos de acesso, com o intuito de uniformizar a distância de amostragem; este procedimento de coleta foi repetido no final do ciclo da cultura, ou seja, aos 96 dias após a semeadura (DAS), para determinação das características químicas do solo. É importante salientar que o acréscimo de 3 tubos de acesso no $S_{2}$ foi devido a irregularidades de alguns canteiros, não sendo possível manter uma malha regular de $5 \times 5 \mathrm{~m}$.

Mediu-se a condutividade elétrica do extrato de saturação $\left(\mathrm{CE}_{\mathrm{es}}\right)$ e se determinaram os cátions solúveis, de acordo com Richards (1954), e estimou-se a relação de adsorção de sódio (RAS). Os cátions trocáveis foram extraídos 
com acetato de amônio 1 mol L-1 (Thomas, 1982), dosando-se o cálcio e o magnésio por espectrofotometria de absorção atômica e o sódio e o potássio por fotometria de emissão de chama. A percentagem de sódio trocável (PST) foi calculada segundo Richards (1954).

Aos 23 DAS se efetuou um desbaste e se adicionou cobertura morta ao $\mathrm{S}_{2}$ com restolhos triturados e desidratados de plantas de feijão com caule, folhas e casca de vagens, aplicando-se $9.000 \mathrm{~kg} \mathrm{ha}^{-1}$; aos 40 DAS se realizou uma adubação de cobertura com $40 \mathrm{~kg} \mathrm{ha}^{-1}$ de $\mathrm{N}$ e $30 \mathrm{~kg} \mathrm{ha}^{-1}$ de $\mathrm{K}_{2} \mathrm{O}$, seguindo-se a recomendação da adubação em fundação.

Após as coletas, as amostras de solo foram secadas ao ar, destorroadas e passadas em malha de $2 \mathrm{~mm}$, para serem procedidas as análises físicas e químicas. Para a determinação das frações granulométricas antes do cultivo da cenoura, adotou-se o método da pipeta, descrito pela EMBRAPA (1997), cujos valores médios se encontram na Tabela 1.

Tabela 1. Médias da composição granulométrica dos dois setores estudados nas camadas de 0-20 e 20-40 cm

\begin{tabular}{lrrrrrr}
\hline \multirow{2}{*}{$\begin{array}{c}\text { Setores } \\
\text { cm }\end{array}$} & \multirow{2}{*}{ Argila } & \multirow{2}{*}{ Silte } & \multicolumn{3}{c}{ Areia } & \\
\cline { 2 - 6 } & & & Grossa & Fina & Total & Classe textural \\
$\mathrm{S}_{1}(0-20)$ & 149,39 & 515,68 & 61,16 & 273,77 & 334,93 & Franco siltoso \\
$\mathrm{S}_{1}(20-40)$ & 169,79 & 492,02 & 74,36 & 271,02 & 345,38 & Franco \\
$\mathrm{S}_{2}(0-20)$ & 124,04 & 340,17 & 101,54 & 434,24 & 535,78 & Franco \\
$\mathrm{S}_{2}(20-40)$ & 87,02 & 391,22 & 135,82 & 385,94 & 521,76 & Franco \\
\hline
\end{tabular}

A água de irrigação foi analisada para os teores de $\mathrm{Ca}^{2+}$, $\mathrm{Mg}^{2+}, \mathrm{Na}^{+}, \mathrm{K}^{+}, \mathrm{pH}$, condutividade elétrica $\left(\mathrm{CE}_{\mathrm{a}}\right)$ e RAS, conforme pode ser visto na Tabela 2.

Tabela 2. Análise química da água usada na irrigação

\begin{tabular}{|c|c|c|c|c|c|c|}
\hline $\mathrm{Ca}^{2+}$ & $\mathrm{Mg}^{2+}$ & $\mathrm{Na}^{+}$ & $\mathrm{K}^{+}$ & \multirow{2}{*}{$\begin{array}{c}\text { CE a } 25{ }^{\circ} \mathrm{C} \\
\text { dS } \mathrm{m}^{-1}\end{array}$} & \multirow{2}{*}{$\mathrm{pH}$} & \multirow{2}{*}{$\begin{array}{c}\text { RAS } \\
\left(\mathrm{mmol} \mathrm{L}^{-1}\right)^{0,5}\end{array}$} \\
\hline \multicolumn{4}{|c|}{$\mathrm{mmol}_{\mathrm{c}} \mathrm{L}^{-1}$} & & & \\
\hline 1,00 & 0,87 & 10,38 & 0,37 & 1,42 & 7,44 & 10,72 \\
\hline
\end{tabular}

O suprimento de água da cultura foi baseado na sua necessidade hídrica. Calculou-se a necessidade de lixiviação ou fração de lixiviação através da equação proposta por Rhoades (1974). Para o presente estudo se adotou uma fração de lixiviação equivalente a $50 \%$ do valor fornecido pela equação citada anteriormente, o que correspondeu a 0,20. Adotaram-se coeficientes de cultivo para cada fase de desenvolvimento, conforme Doorenbos \& Pruitt (1977).

Ao final do ciclo da cultura foi efetuada a colheita nos dois setores de irrigação com o intuito de relacionar a salinidade do solo e o manejo adotado no experimento com a produtividade da cultura. Esta foi estimada pela área ocupada por 343 plantas no $\mathrm{S}_{1}$ e 364 plantas no $\mathrm{S}_{2}$, já que ao redor de cada tubo de acesso (49 no $S_{1}$ e 52 no $S_{2}$ ) foram colhidas 7 plantas.

Realizou-se a análise de estatística descritiva através da avaliação de medidas de tendência central e dispersão e de aderência à distribuição Normal, segundo o teste de Shapiro \& Wilk (1965). Para a análise geoestatística, utilizouse a ferramenta geoestatística GS ${ }^{+}$(Robertson, 1998). Após a obtenção dos semivariogramas experimentais se testaram os modelos gaussiano, esférico e exponencial, efetuandose a validação cruzada segundo a técnica de Jack-Knifing (Vauclin et al., 1983). Para confecção dos mapas de isolinhas trabalhou-se com o software Surfer for Windows versão 8.0 (SURFER, 2002).

Com vistas à caracterização do grau de variabilidade, analisaram-se valores de coeficiente de variação (CV) dos atributos, conforme sugerido por Warrick \& Nielsen (1980), os quais consideram baixa variabilidade quando $\mathrm{CV}<12 \%$; média para o intervalo $12-60 \%$ e alta variabilidade quando CV $>60 \%$. No tocante ao grau de dependência espacial, utilizou-se a classificação proposta por Cambardella et al. (1994), na qual um atributo pode ser considerado exibindo forte, moderada ou fraca dependência espacial, em função da relação entre o efeito pepita e o patamar de seu semivariograma ajustado. Valores inferiores a 25\% caracterizam forte dependência espacial, entre 25 e 75\% moderada, enquanto acima de 75\%, fraca dependência. Para as variáveis que apresentaram efeito pepita puro, ou seja, ausência de dependência espacial e aquelas com alcance reduzido, realizou-se o teste de Tukey a nível de 5\% probabilidade, com o intuito de se comparar as médias de tais variáveis entre as duas épocas de amostragem.

\section{RESULTADOS E DISCUSSÃO}

$\mathrm{O}$ resultado da análise estatística descritiva para $\mathrm{CE}_{\mathrm{es}}$, cátions solúveis $\left(\mathrm{Ca}^{2+}, \mathrm{Mg}^{2+}, \mathrm{Na}^{+}\right.$e $\left.\mathrm{K}^{+}\right)$e a RAS para o $\mathrm{S}_{1}$ (0-20 e 20-40 cm) antes e aos 96 DAS, se encontram na Tabela 3. Analisando-se a média das variáveis na camada de 0-20 cm, verifica-se que a $\mathrm{CE}_{\mathrm{es}}$ se manteve praticamente constante, após o cultivo de cenoura, enquanto os elementos $\mathrm{Ca}^{2+}$ e $\mathrm{Na}^{+}$apresentaram teores mais elevados ao final do ciclo da cenoura. Para os teores de $\mathrm{Mg}^{2+}$ e $\mathrm{K}^{+}$se observaram decréscimos de 31,48 e 52,38\%, respectivamente, o que pode ter sido ocasionado, provavelmente por lixiviação e, ainda, absorção desses elementos pelas plantas. Uma provável explicação para a estabilidade do valor da $\mathrm{CE}_{\mathrm{es}}$ está associada principalmente ao manejo da irrigação, em que o turno de rega foi diário, da adoção de uma fração de lixiviação de 0,20, absorção pela cultura, precipitação química e da ocorrência de eventos pluviométricos durante o período de execução do experimento. A RAS, após 96 DAS, apresentou um aumento de 9,00 para $11,45\left(\operatorname{mmol}_{\mathrm{C}} \mathrm{L}^{-1}\right)^{0,5}$, ocasionado pelo acréscimo do teor de sódio e diminuição no teor de $\mathrm{Mg}^{2+}$.

Quanto aos coeficientes de variação, os maiores valores foram encontrados para o $\mathrm{K}^{+}$, resultados esses comumente encontrados na literatura; as demais variáveis apresentaram média variabilidade pelo critério de Warrick \& Nielsen (1980), com valores de CV na faixa de 12 a 60\%. Com respeito ao teste de Normalidade, as variáveis que apresentaram aderência a tal distribuição, foram $\mathrm{Na}^{+}$e RAS (antes da semeadura), $\mathrm{CE}_{\mathrm{es}}, \mathrm{Ca}^{2+}$ e RAS (96 DAS).

Na camada de 20-40 cm, a $\mathrm{CE}_{\mathrm{es}}$ aumentou em 0,58 dS m-1 e a RAS passou de 6,49 para 7,93 $\left(\mathrm{mmol}_{\mathrm{C}} \mathrm{L}^{-1}\right)^{0,5}$, sendo observados, também, pequenos incrementos nos teores de $\mathrm{Ca}^{2+}$ e $\mathrm{Na}^{+}$e redução nos de $\mathrm{Mg}^{2+}$ e $\mathrm{K}^{+}$. Os valores de $\mathrm{CV}$ mostraram o 
mesmo comportamento da camada 0-20 cm. O aumento das RAS após o cultivo seguiu o mesmo padrão de acréscimo da primeira camada avaliada, em virtude do $\mathrm{Na}^{+}$também ter aumentado, o que pode ser devido à predominância do sódio na água utilizada para irrigação (Tabela 2), sendo fator preocupante, por contribuir para a sodificação dos solos e degradação de suas propriedades físicas (Freire et al., 2003a e b).

No $S_{2}$ (Tabela 4), a $\mathrm{CE}_{\mathrm{es}}$ decresceu nas duas camadas ao final do ciclo, enquanto os valores das RAS foram mais elevados, em virtude do decréscimo de $\mathrm{Ca}^{2+}$ e $\mathrm{Mg}^{2+}$ da solução do solo, aumentando a concentração relativa de $\mathrm{Na}^{+}$. Os valores de RAS se apresentaram aderentes à distribuição Normal nas duas épocas e camadas.
Pode-se afirmar que, após o ciclo da cenoura, não houve alterações relevantes nos valores de $\mathrm{CE}_{\mathrm{es}}$, cátions solúveis e RAS para ambos os setores, o que, de certa forma, pode ser justificado pelo manejo da irrigação, uma vez que o fornecimento de água foi baseado na necessidade da cultura e ainda se adotou uma fração de lixiviação de 0,20 . Blanco \& Folegatti (2002), trabalhando com pepino em estufa e adotando três níveis de salinidade de água de irrigação, notaram que a lâmina correspondente a 1,25 da evapotranspiração da cultura não foi suficiente para reduzir a salinidade do solo. Salienta-se que estudos mais completos devem ser efetuados no que concerne à profundidade de avaliação da salinidade que, para o presente trabalho, se limitou a $40 \mathrm{~cm}$,

Tabela 3. Estatística descritiva para $\mathrm{CE}_{\mathrm{es}}\left(\mathrm{dS} \mathrm{m} \mathrm{m}^{-1}\right), \mathrm{Ca}, \mathrm{Mg}$, $\mathrm{Na}$ e K $\left(\mathrm{cmol}_{\mathrm{C}} \mathrm{L}^{-1}\right)$ e RAS (mmol L-1) $)^{0.5}$, do $\mathrm{S}_{1}$, nas duas camadas, antes do plantio e $96 \mathrm{DAS}$

\begin{tabular}{|c|c|c|c|c|c|c|c|c|c|c|c|c|}
\hline \multirow{2}{*}{ Par. } & \multicolumn{6}{|c|}{ Antes da semeadura } & \multicolumn{6}{|c|}{96 DAS } \\
\hline & $\mathrm{CE}_{\mathrm{es}}$ & $\mathrm{Ca}^{2+}$ & $\mathrm{Mg}^{2+}$ & $\mathrm{Na}^{+}$ & $\mathrm{K}^{+}$ & RAS & $\mathrm{CE}_{\mathrm{es}}$ & $\mathrm{Ca}^{2+}$ & $\mathrm{Mg}^{2+}$ & $\mathrm{Na}^{+}$ & $\mathrm{K}^{+}$ & RAS \\
\hline \multicolumn{13}{|c|}{ Setor $1(0-20 \mathrm{~cm})$} \\
\hline M & 3,62 & 0,55 & 0,54 & 2,04 & 0,21 & 9,00 & 3,60 & 0,60 & 0,37 & 2,52 & 0,10 & 11,45 \\
\hline D.P. & 0,86 & 0,18 & 0,24 & 0,43 & 0,16 & 1,58 & 1,26 & 0,19 & 0,16 & 0,96 & 0,08 & 2,19 \\
\hline C.V. & 23,59 & 33,55 & 45,67 & 21,25 & 76,32 & 17,55 & 35,12 & 30,92 & 43,22 & 38,22 & 79,18 & 19,14 \\
\hline A & 1,12 & 1,00 & 1,10 & 0,66 & 1,75 & $-0,15$ & 0,38 & 0,62 & 1,46 & 1,35 & 1,06 & 0,29 \\
\hline C & 0,86 & 0,47 & 1,51 & 0,80 & 4,30 & $-0,57$ & $-1,01$ & 0,51 & 2,16 & 3,60 & 0,90 & 0,01 \\
\hline N.A. & 43 & 43 & 43 & 43 & 43 & 43 & 43 & 42 & 43 & 43 & 43 & 41 \\
\hline W & 0,90 & 0,91 & 0,86 & $0,96^{\mathrm{ns}}$ & 0,85 & $0,98 \mathrm{~ns}$ & $0,93^{\mathrm{ns}}$ & $0,95^{\mathrm{ns}}$ & 0,86 & 0,90 & 0,90 & $0,97 \mathrm{~ns}$ \\
\hline $\mathrm{P}<\mathrm{W}$ & 0,00 & 0,00 & 0,00 & 0,14 & 0,00 & 0,55 & 0,01 & 0,79 & 0,00 & 0,00 & 0,00 & 0,39 \\
\hline \multicolumn{13}{|c|}{ Setor $1(20-40 \mathrm{~cm})$} \\
\hline M & 2,50 & 0,44 & 0,49 & 1,36 & 0,19 & 6,49 & 3,08 & 0,53 & 0,42 & 1,73 & 0,13 & 7,93 \\
\hline D.P. & 0,75 & 0,15 & 0,24 & 0,44 & 0,18 & 1,85 & 1,32 & 0,25 & 0,31 & 0,69 & 0,11 & 1,60 \\
\hline C.V. & 29,88 & 34,32 & 48,45 & 32,24 & 94,21 & 28,44 & 42,81 & 46,73 & 74,15 & 40,17 & 88,96 & 20,16 \\
\hline A & 1,44 & 0,73 & 1,62 & 0,45 & 0,96 & 0,32 & 0,42 & 0,81 & 1,12 & 0,22 & 0,876 & 0,01 \\
\hline C & 4,44 & 0,48 & 1,87 & 0,24 & $-0,16$ & $-0,34$ & $-1,17$ & $-0,26$ & 0,80 & $-1,43$ & 0,134 & $-0,73$ \\
\hline N.A. & 42 & 42 & 39 & 42 & 42 & 42 & 42 & 42 & 42 & 42 & 42 & 40 \\
\hline W & 0,91 & $0,95^{\mathrm{ns}}$ & 0,79 & $0,97^{\mathrm{ns}}$ & 0,86 & 0,98 ns & 0,92 & 0,90 & 0,89 ns & 0,91 & 0,90 & $0,98 \mathrm{~ns}$ \\
\hline $\mathrm{P}<\mathrm{W}$ & 0,00 & 0,08 & 0,00 & 0,22 & 0,00 & 0,64 & 0,00 & 0,00 & 0,01 & 0,00 & 0,001 & 0,66 \\
\hline
\end{tabular}

Par: Parâmetros; M: média; C.V.: coeficiente de variação; A: assimetria; C: curtose; N.A.: número de amostras; W: valor calculado do Teste Shapiro-Wilk. ns não significativo = Distribuição Normal

Tabela 4. Estatística descritiva para a $\mathrm{CE}_{\mathrm{es}}\left(\mathrm{dS} \mathrm{m}^{-1}\right), \mathrm{Ca}^{2+}, \mathrm{Mg}^{2+}, \mathrm{Na}^{+}$e K$^{+}\left(\mathrm{cmol}_{\mathrm{c}} \mathrm{L}^{-1}\right)$ e RAS (mmol L-1 $)^{0,5}$ do $\mathrm{S}_{2}$ nas duas camadas, antes do plantio e $96 \mathrm{DAS}$

\begin{tabular}{|c|c|c|c|c|c|c|c|c|c|c|c|c|}
\hline \multirow{2}{*}{ Par. } & \multicolumn{6}{|c|}{ Antes da semeadura } & \multicolumn{6}{|c|}{96 DAS } \\
\hline & $\mathrm{CE}_{\mathrm{es}}$ & $\mathrm{Ca}^{2+}$ & $\mathrm{Mg}^{2+}$ & $\mathrm{Na}^{+}$ & $\mathrm{K}^{+}$ & RAS & $\mathrm{CE}_{\mathrm{es}}$ & $\mathrm{Ca}^{2+}$ & $\mathrm{Mg}^{2+}$ & $\mathrm{Na}^{+}$ & $\mathrm{K}^{+}$ & RAS \\
\hline \multicolumn{13}{|c|}{ Setor $2(0-20 \mathrm{~cm})$} \\
\hline M & 3,51 & 0,55 & 0,51 & 1,90 & 0,11 & 8,41 & 2,91 & 0,35 & 0,31 & 1,84 & 0,003 & 10,22 \\
\hline D.P. & 0,94 & 0,20 & 0,23 & 0,46 & 0,08 & 1,42 & 1,45 & 0,19 & 0,19 & 0,72 & 0,004 & 1,86 \\
\hline C.V. & 26,63 & 35,74 & 45,80 & 24,40 & 68,31 & 16,91 & 49,64 & 54,83 & 61,94 & 39,16 & 143,0 & 18,17 \\
\hline A & 0,71 & 1,26 & 1,71 & 0,60 & 1,77 & 0,21 & 2,24 & 3,03 & 3,30 & 1,68 & 3,90 & $-0,29$ \\
\hline C & 0,44 & 1,96 & 3,90 & 0,65 & 3,09 & 0,80 & 2,39 & 12,35 & 14,26 & 3,48 & 14,89 & $-0,41$ \\
\hline N.A. & 36 & 36 & 36 & 36 & 36 & 36 & 36 & 36 & 36 & 36 & 36 & 36 \\
\hline W & $0,96^{\mathrm{ns}}$ & 0,90 & 0,85 & $0,95^{\mathrm{ns}}$ & 0,80 & $0,98^{\mathrm{ns}}$ & 0,74 & 0,71 & 0,68 & 0,88 & 0,41 & 0,97 ns \\
\hline $\mathrm{P}<\mathrm{W}$ & 0,15 & 0,00 & 0,00 & 0,15 & 0,00 & 0,72 & 0,00 & 0,00 & 0,00 & 0,00 & 0,00 & 0,50 \\
\hline \multicolumn{13}{|c|}{ Setor $2(20-40 \mathrm{~cm})$} \\
\hline M & 2,90 & 0,48 & 0,40 & 1,54 & 0,06 & 7,46 & 2,53 & 0,45 & 0,31 & 1,62 & 0,001 & 8,46 \\
\hline D.P. & 0,59 & 0,14 & 0,14 & 0,29 & 0,04 & 1,14 & 1,78 & 0,28 & 0,25 & 0,65 & 0,001 & 1,20 \\
\hline C.V. & 20,37 & 28,36 & 35,89 & 19,11 & 72,13 & 15,33 & 46,62 & 62,30 & 81,29 & 40,54 & 91,83 & 14,14 \\
\hline A & 0,19 & 0,22 & 0,83 & 0,25 & 1,59 & $-0,16$ & 1,77 & 2,12 & 2,22 & 1,48 & 1,960 & 0,34 \\
\hline C & $-1,21$ & $-0,89$ & 0,33 & $-0,92$ & 3,58 & $-0,97$ & 2,23 & 4,11 & 4,38 & 1,19 & 4,540 & 0,58 \\
\hline N.A. & 43 & 43 & 43 & 43 & 40 & 43 & 43 & 43 & 43 & 43 & 42 & 43 \\
\hline W & $0,95^{\mathrm{ns}}$ & $0,97 \mathrm{~ns}$ & $0,94^{\mathrm{ns}}$ & $0,95^{\mathrm{ns}}$ & 0,87 & $0,96^{\mathrm{ns}}$ & 0,74 & 0,71 & 0,67 & 0,79 & 0,790 & 0,97 ns \\
\hline $\mathrm{P}<\mathrm{W}$ & 0,05 & 0,30 & 0,03 & 0,05 & 0,00 & 0,21 & 0,00 & 0,00 & 0,00 & 0,00 & 0,000 & 0,23 \\
\hline
\end{tabular}


em razão de ser a profundidade média explorada pela cultura da cenoura.

Um fato importante digno de realce é que a $\mathrm{CE}_{\mathrm{es}}$ e a RAS se mantiveram sempre em valores mais elevados na camada superficial do solo $(0-20 \mathrm{~cm})$, possivelmente em razão da evaporação superficial e da absorção pelo sistema radicular; este fato também foi observado por Dias et al. (2004), trabalhando com melão e utilizando águas de irrigação com três níveis de salinidade (1,2, 2,5 e 4,5 dS m-1), os autores verificaram que à camada de $0-15 \mathrm{~cm}$ exibiu os maiores valores de $\mathrm{CE}_{\mathrm{es}}$, quando comparada com as camadas de 15-30 e 30-45 cm. Blanco \& Folegatti (2002), trabalhando com pepino em estufa, também constataram comportamento semelhante.

Com relação aos cátions trocáveis verifica-se, para todos os elementos, uma redução de valores ao final do ciclo da cultura e, conseqüentemente, a redução da PST, na ordem de 0,78 unidades, atingindo o valor de $10,36 \%$ na camada de 0-20 cm, no $S_{1}$ (Tabela 5). Segundo a classificação proposta por Richards (1954), este solo não seria classificado sódico, uma vez que o valor de PST não ultrapassou 15\%, embora para determinados autores, este valor já pode estar interferindo nas propriedades físicas do solo (Freire, 2001). Todos os CV's caracterizaram média variabilidade.

Os valores de PST para a camada de $20-40 \mathrm{~cm}$ foram inferiores aos da camada superior, tendo ocorrido um acréscimo de 5,89 para 8,74\% durante o ciclo.

Para o $\mathrm{S}_{2}$, os valores de PST foram aumentados durante o ciclo, em virtude do aumento de $\mathrm{Na}$ no complexo de troca para as duas camadas (Tabela 6). Ao se comparar o $S_{1}$ e $S_{2}$, nota-se que este último apresentou menores valores de PST, o que pode ser explicado pelos teores elevados de areia no $\mathrm{S}_{2}$, para as duas camadas (Tabela 1).

Na Tabela 7 estão expressos os parâmetros dos semivariogramas para $\mathrm{CE}_{\mathrm{es}}$, RAS e PST, nos dois setores e nas duas camadas. A validação para todos os semivariogramas pela técnica de Jack Knifing apresentou erros padronizados com média próximo a zero e desvio-padrão próximo à unidade. Percebe-se que, para o $S_{1}$, nas duas camadas e épocas de amostragem, a $\mathrm{CE}_{\mathrm{es}}$ apresentou efeito pepita puro indicando ausência de dependência espacial, ou seja, o uso da estatística clássica seria adequado; para o $\mathrm{S}_{2}$, a $\mathrm{CE}_{\mathrm{es}}$ apresentou efeito pepita puro somente para a camada de $0-20 \mathrm{~cm}$ e na $2^{a}$ coleta, embora os alcances encontrados para as demais camadas tenham sido relativamente baixos, variando de 7,95 a 11,00 m, o que indica a distância a partir da qual as amostras são consideradas independentes.

Comportamento semelhante ao da $\mathrm{CE}_{\mathrm{es}}$ foi observado para a RAS, tendo predominado ausência de dependência espacial, mesmo que tal dependência tenha sido observada para a primeira coleta do $S_{1}(0-20 \mathrm{~cm})$, e para a camada de $20-40 \mathrm{~cm}$ no $\mathrm{S}_{2}$, para as duas coletas. Este comportamento de ausência de dependência espacial pode estar associado ao tipo de variável uma vez que tanto a CEes como a RAS são medidas a partir de extrato de saturação do solo, podendo ocorrer variações no método de preparo da pasta saturada.

Com relação à PST, observa-se comportamento diferenciado uma vez que, para ambos os setores, camadas e épocas de amostragem, esta variável apresentou dependência espacial, exceto para a camada de $0-20 \mathrm{~cm}$ e na segunda coleta do $S_{1}$, comportamento que pode estar relacionado com a estabilidade que a fração trocável tende a manter ao longo do tempo.

Como forma de validar as diferenças ocorridas entre as duas épocas de amostragem para os dois setores, realizou-se o teste de Tukey para as variáveis $\mathrm{CE}_{\mathrm{es}}$, RAS e PST (Tabela 8); para tal, foram utilizados apenas valores obtidos em amostras coletadas a distâncias maiores que o alcance, visando manter a característica de independência entre as

Tabela 5. Estatística descritiva para o $\mathrm{Ca}, \mathrm{Mg}, \mathrm{Na}, \mathrm{K}$ trocáveis $\left(\mathrm{cmol}_{\mathrm{c}} \mathrm{kg}^{-1}\right)$ e PST do $\mathrm{S}_{1}$, nas duas camadas, antes do plantio e aos 96 DAS

\begin{tabular}{|c|c|c|c|c|c|c|c|c|c|c|}
\hline \multirow{2}{*}{ Par. } & \multicolumn{5}{|c|}{ Antes da semeadura } & \multicolumn{5}{|c|}{96 DAS } \\
\hline & $\mathrm{Ca}$ & $\mathrm{Mg}$ & $\mathrm{Na}$ & K & PST & $\mathrm{Ca}$ & $\mathrm{Mg}$ & $\mathrm{Na}$ & K & PST \\
\hline \multicolumn{11}{|c|}{ Setor $1(0-20 \mathrm{~cm})$} \\
\hline M & 8,11 & 3,80 & 1,58 & 0,99 & 11,14 & 7,78 & 3,68 & 1,38 & 0,60 & 10,36 \\
\hline D.P. & 1,14 & 0,61 & 0,28 & 0,48 & 2,15 & 1,26 & 0,65 & 0,25 & 0,35 & 1,97 \\
\hline C.V. & 13,74 & 16,00 & 17,58 & 47,96 & 19,27 & 16,15 & 17,66 & 17,95 & 58,03 & 19,00 \\
\hline$A$ & 0,21 & $-0,21$ & 0,47 & 0,29 & 0,11 & 0,13 & $-0,07$ & $-0,32$ & 0,90 & 0,64 \\
\hline C & $-0,61$ & $-0,52$ & $-0,007$ & $-1,11$ & $-0,78$ & $-0,46$ & $-0,87$ & $-0,13$ & 0,14 & 0,82 \\
\hline N.A. & 45 & 45 & 45 & 45 & 48,00 & 45 & 45 & 45 & 45 & 49,00 \\
\hline W & $0,98^{\mathrm{ns}}$ & $0,98^{\mathrm{ns}}$ & $0,97^{\mathrm{ns}}$ & $0,94^{\mathrm{ns}}$ & $0,97^{\mathrm{ns}}$ & $0,98^{\mathrm{ns}}$ & $0,97^{\mathrm{ns}}$ & $0,98^{\mathrm{ns}}$ & 0,91 & $0,96^{\mathrm{ns}}$ \\
\hline $\mathrm{P}<\mathrm{W}$ & 0,55 & 0,77 & 0,20 & 0,02 & 0,23 & 0,57 & 0,40 & 0,43 & 0,00 & 0,08 \\
\hline \multicolumn{11}{|c|}{ Setor $1(20-40 \mathrm{~cm})$} \\
\hline M & 9,20 & 4,36 & 0,90 & 0,79 & 5,89 & 6,91 & 3,24 & 1,00 & 0,64 & 8,74 \\
\hline D.P. & 1,19 & 0,68 & 0,27 & 0,63 & 1,78 & 0,99 & 0,52 & 0,30 & 0,52 & 1,83 \\
\hline C.V. & 12,90 & 15,61 & 29,65 & 80,12 & 30,22 & 14,34 & 16,04 & 29,54 & 79,97 & 20,94 \\
\hline C & $-0,08$ & $-0,07$ & $-1,48$ & $-0,24$ & $-1,16$ & 0,98 & 0,54 & 3,60 & 0,54 & 0,30 \\
\hline N.A. & 41 & 41 & 47 & 41 & 47 & 41 & 41 & 47 & 41 & 45 \\
\hline W & $0,98 \mathrm{~ns}$ & $0,98 \mathrm{~ns}$ & 0,92 & 0,88 & $0,95^{\mathrm{ns}}$ & $0,98 \mathrm{~ns}$ & $0,94^{\mathrm{ns}}$ & 0,93 & 0,89 & $0,97 \mathrm{~ns}$ \\
\hline$P<W$ & 0,77 & 0,69 & 0,00 & 0,00 & 0,05 & 0,49 & 0,04 & 0,00 & 0,00 & 0,24 \\
\hline
\end{tabular}


Tabela 6. Estatística descritiva para o $\mathrm{Ca}, \mathrm{Mg}, \mathrm{Na}$, $\mathrm{K}$ trocáveis $\left(\mathrm{cmol}_{\mathrm{c}} \mathrm{Kg}^{-1}\right)$ e PST do $\mathrm{S}$, nas duas camadas, antes do plantio e aos 96 DAS

\begin{tabular}{|c|c|c|c|c|c|c|c|c|c|c|}
\hline \multirow{2}{*}{ Par. } & \multicolumn{5}{|c|}{ Antes da semeadura } & \multicolumn{5}{|c|}{96 DAS } \\
\hline & $\mathrm{Ca}$ & $\mathrm{Mg}$ & $\mathrm{Na}$ & K & PST & $\mathrm{Ca}$ & $\mathrm{Mg}$ & $\mathrm{Na}$ & K & PST \\
\hline \multicolumn{11}{|c|}{ Setor $2(0-20 \mathrm{~cm})$} \\
\hline M & 5,82 & 2,41 & 0,71 & 0,41 & 7,59 & 4,91 & 1,69 & 0,75 & 0,34 & 9,74 \\
\hline D.P. & 0,78 & 0,40 & 0,23 & 0,14 & 2,17 & 0,77 & 0,37 & 0,21 & 0,20 & 2,33 \\
\hline C.V. & 13,32 & 16,75 & 31,73 & 32,92 & 28,55 & 15,73 & 21,81 & 28,11 & 58,37 & 23,94 \\
\hline$A$ & $-0,25$ & 0,30 & 0,50 & 1,00 & 0,80 & $-0,18$ & 0,30 & 0,57 & 3,51 & 0,18 \\
\hline C & $-0,53$ & $-0,58$ & $-0,92$ & 2,07 & 1,10 & $-0,18$ & $-0,58$ & $-0,47$ & 13,81 & $-0,68$ \\
\hline N.A. & 45 & 45 & 45 & 45 & 46 & 45 & 45 & 45 & 45 & 50 \\
\hline w & $0,97^{\mathrm{ns}}$ & $0,98^{\mathrm{ns}}$ & 0,93 & $0,94^{\mathrm{ns}}$ & $0,95^{\mathrm{ns}}$ & $0,99 \mathrm{~ns}$ & $0,97^{\mathrm{ns}}$ & $0,94^{\mathrm{ns}}$ & 0,59 & $0,98 \mathrm{~ns}$ \\
\hline$P<W$ & 0,41 & 0,43 & 0,00 & 0,01 & 0,05 & 0,83 & 0,30 & 0,02 & 0,00 & 0,61 \\
\hline \multicolumn{11}{|c|}{ Setor $2(20-40 \mathrm{~cm})$} \\
\hline M & 5,87 & 2,24 & 0,53 & 0,23 & 5,94 & 5,43 & 1,87 & 0,65 & 0,24 & 8,51 \\
\hline D.P. & 0,86 & 0,37 & 0,18 & 0,09 & 1,62 & 1,14 & 0,34 & 0,02 & 0,006 & 2,45 \\
\hline C.V. & 14,60 & 16,62 & 34,61 & 37,74 & 27,35 & 21,01 & 31,26 & 22,38 & 34,27 & 28,80 \\
\hline$A$ & 0,59 & 0,10 & 1,08 & 0,41 & 0,70 & $-0,60$ & $-0,35$ & 0,60 & 0,82 & 0,71 \\
\hline C & 3,48 & 1,76 & 1,48 & $-0,43$ & 0,72 & $-0,63$ & $-1,25$ & $-0,03$ & 0,87 & $-0,06$ \\
\hline N.A. & 46 & 46 & 46 & 46 & 47,00 & 46 & 46 & 46 & 46 & 51 \\
\hline w & 0,96 ns & 0,97 ns & 0,92 & $0,96^{\mathrm{ns}}$ & $0,96 \mathrm{~ns}$ & $0,94 \mathrm{~ns}$ & 0,92 & $0,96^{\mathrm{ns}}$ & $0,95 \mathrm{~ns}$ & $0,95 \mathrm{~ns}$ \\
\hline$P<W$ & 0,12 & 0,31 & 0,00 & 0,09 & 0,15 & 0,02 & 0,00 & 0,15 & 0,05 & 0,04 \\
\hline
\end{tabular}

Tabela 7. Parâmetros dos semivariogramas da $\mathrm{CE}_{\mathrm{es}}$ RAS e PST nos dois setores, camadas e épocas de amostragem

\begin{tabular}{|c|c|c|c|c|c|c|}
\hline \multirow[t]{2}{*}{$\begin{array}{l}\text { Setores e } \\
\text { camadas }\end{array}$} & Modelo & $\mathrm{C}_{0}$ & $C_{0}+C_{1}$ & $A(m)$ & GD (\%) & $\mathbf{R}^{2}$ \\
\hline & \multicolumn{6}{|c|}{ CEes } \\
\hline \multicolumn{7}{|l|}{ Setor 1} \\
\hline 0-20 1a Coleta & EPP & - & - & - & - & - \\
\hline 0-20 2a Coleta & EPP & - & - & - & - & - \\
\hline 20-40 1’ Coleta & EPP & - & - & - & - & - \\
\hline 20-40 2ª Coleta & EPP & - & - & - & - & - \\
\hline \multicolumn{7}{|l|}{ Setor 2} \\
\hline 0-20 1a Coleta & EXP & 0,256 & 0,891 & 10,170 & 28,732 & 0,953 \\
\hline 0-20 2a Coleta & EPP & - & - & - & - & - \\
\hline 20-40 1’ Coleta & ESF & 0,075 & 0,364 & 7,9500 & 20,604 & 0,801 \\
\hline \multirow[t]{2}{*}{ 20-40 2ª Coleta } & ESF & 0,001 & 1,680 & 11,000 & 0,0600 & 0,896 \\
\hline & \multicolumn{6}{|c|}{ RAS } \\
\hline \multicolumn{7}{|l|}{ Setor 1} \\
\hline 0-20 1a Coleta & ESF & 0,445 & 2,947 & 19,97 & 15,100 & 0,882 \\
\hline 0-20 2 Coleta & EPP & - & - & - & - & - \\
\hline 20-40 1’a Coleta & EPP & - & - & - & - & - \\
\hline 20-40 2ª Coleta & EPP & - & - & - & - & - \\
\hline \multicolumn{7}{|l|}{ Setor 2} \\
\hline 0-20 1a Coleta & EPP & - & - & - & - & - \\
\hline 0-20 2ª Coleta & EPP & - & - & - & - & - \\
\hline 20-40 1 Coleta & ESF & 0,706 & 1,413 & 17,750 & 49,965 & 0,986 \\
\hline \multirow[t]{2}{*}{ 20-40 2ª Coleta } & ESF & 0,001 & 1,542 & 9,770 & 0,0649 & 0,836 \\
\hline & \multicolumn{6}{|c|}{ PST } \\
\hline \multicolumn{7}{|l|}{ Setor 1} \\
\hline 0-20 1a Coleta & ESF & 0,980 & 5,273 & 20,270 & 18,590 & 0,951 \\
\hline 0-20 2a Coleta & EPP & - & - & - & - & - \\
\hline 20-40 1’ Coleta & ESF & 0,954 & 3,815 & 23,940 & 25,010 & 0,950 \\
\hline 20-40 2ª Coleta & ESF & 1,387 & 4,303 & 19,200 & 32,230 & 0,978 \\
\hline \multicolumn{7}{|l|}{ Setor 2} \\
\hline 0-20 1a Coleta & ESF & 2,430 & 5,480 & 33,910 & 44,343 & 0,824 \\
\hline 0-20 2 $2^{a}$ Coleta & ESF & 0,970 & 5,273 & 7,670 & 18,396 & 0,719 \\
\hline 20-40 1 Coleta & EXP & 0,444 & 2,465 & 33,130 & 18,012 & 0,556 \\
\hline 20-40 2ª Coleta & EPP & - & - & - & - & - \\
\hline
\end{tabular}

1 a coleta $=$ antes da semeadura; $2^{a}$ coleta $=96 \mathrm{DAS} ; \mathrm{GD}=$ Grau de dependência de Cambardella; $\mathrm{EPP}=$ efeito pepita puro; $\mathrm{ESF}=$ modelo esférico; $\mathrm{EXP}=$ modelo exponencial; $\mathrm{C}_{0}=$ Efeito pepita; $\mathrm{C}_{0}+\mathrm{C}_{1}=$ Patamar; $\mathrm{A}=$ Alcance amostras. Com relação à $\mathrm{CE}_{\mathrm{es}}$, os dois setores e respectivas camadas não diferiram estatisticamente, exceto o $\mathrm{S}_{1}$ $(20-40 \mathrm{~cm})$, em que a segunda coleta de solo apresentou média estatisticamente diferente da primeira coleta; para a RAS, no entanto, houve aumento significativo para ambos os setores e camadas, exceto para o $\mathrm{S}_{2}(20-40 \mathrm{~cm})$; este acréscimo pode ter ocorrido provavelmente pela contínua adição do íon sódio via água de irrigação.

Para a PST só se efetuou o teste de média no $\mathrm{S}_{1}$, uma vez que no $S_{2}$ foram encontrados alcances altos, inviabilizandose a escolha de amostras independentes. Verifica-se que o $S_{1}$ $(20-40 \mathrm{~cm})$ apresentou valor médio de PST ao final do ciclo estatisticamente superior à primeira coleta. Pode-se observar claramente que houve movimentação de sais da camada de 0-20 $\mathrm{cm}$ do $S_{1}$ para a camada de $20-40 \mathrm{~cm}$, uma vez que todas as variáveis avaliadas indicaram valores estatisticamente superiores no final do ciclo para o $S_{1}(20-40 \mathrm{~cm})$, quando se compara com os valores do início do ciclo.

Visando observar a distribuição espacial da $\mathrm{CE}_{\mathrm{es}}$, mapas de isolinhas foram confeccionados adotando-se a técnica da krigagem, quando foi possível o ajuste de semivariogramas, e se utilizando a interpolação através do inverso do quadrado da distância, para as variáveis que mostraram efeito

Tabela 8. Teste de Tukey a nível de $5 \%$ de probabilidade para a $\mathrm{CE}_{\text {es' }}$ RAS e PST, nos setores de irrigação, camadas e épocas de amostragem

\begin{tabular}{|c|c|c|c|c|c|c|c|c|}
\hline & \multicolumn{2}{|c|}{$S_{1}(0-20)$} & \multicolumn{2}{|c|}{$S_{1}(20-40)$} & \multicolumn{2}{|c|}{$S_{2}(0-20)$} & \multicolumn{2}{|c|}{$S_{2}(20-40)$} \\
\hline & $1^{a} \mathrm{C}$ & $2^{\mathrm{a} C \mathrm{C}}$ & $1 \mathrm{aC}$ & $2^{\mathrm{a} C}$ & 1a C & $2^{a} \mathrm{C}$ & $1 \mathrm{a} C$ & $2^{a} \mathrm{C}$ \\
\hline $\mathrm{CE}_{\mathrm{es}}$ & $3,62 a$ & $3,60 a$ & $2,52 \mathrm{~b}$ & $3,08 a$ & $3,57 \mathrm{a}$ & $2,92 \mathrm{a}$ & $3,04 a$ & $2,48 a$ \\
\hline RAS & $9,07 \mathrm{~b}$ & 11,65 a & $6,52 b$ & 7,94 a & $8,41 \mathrm{~b}$ & $10,22 \mathrm{a}$ & $7,77 \mathrm{a}$ & $8,33 \mathrm{a}$ \\
\hline PST & $11,01 \mathrm{a}$ & $10,31 \mathrm{a}$ & $6,19 \mathrm{~b}$ & $8,98 \mathrm{a}$ & * & * & * & * \\
\hline
\end{tabular}

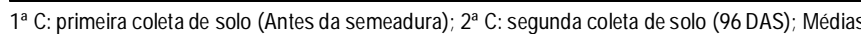
seguidas de mesma letra entre linhas e dentro de cada setor e camada não diferem estatisticamente a nível de $5 \%$ de probabilidade pelo Teste de Tukey; *: não pôde ser realizado, uma vez que não foi encontrado, através dos semivariogramas, o alcance, ou seja, a distância a partir da qual as amostras seriam independentes 
pepita puro. A Figura 1 exibe os mapas para os dois setores e nas duas camadas representando, através das áreas escuras, os locais em que o solo se apresenta salino, ou seja, com $\mathrm{CE}_{\mathrm{es}} \geq 4 \mathrm{dS} \mathrm{m}^{-1}$ de acordo com Richards (1954). O S apresentou áreas salinas de 15,28 e 0,67\% da área total, na primeira coleta de solo para as camadas de 0-20 e 20-40 cm (Figuras 1A e 1C), respectivamente; aos 96 DAS, os valores de $\mathrm{CE}_{\mathrm{es}}$ aumentaram, respectivamente, para 22,77 e $7,40 \%$ (Figuras $1 \mathrm{~B}$ e $1 \mathrm{D}$ ); para o $\mathrm{S}_{2}(0-20$ e 20-40 cm), os valores iniciais foram de 11,94 e $0,00 \%$ (Figuras 1E e 1G) e, no final do ciclo, apresentaram per-
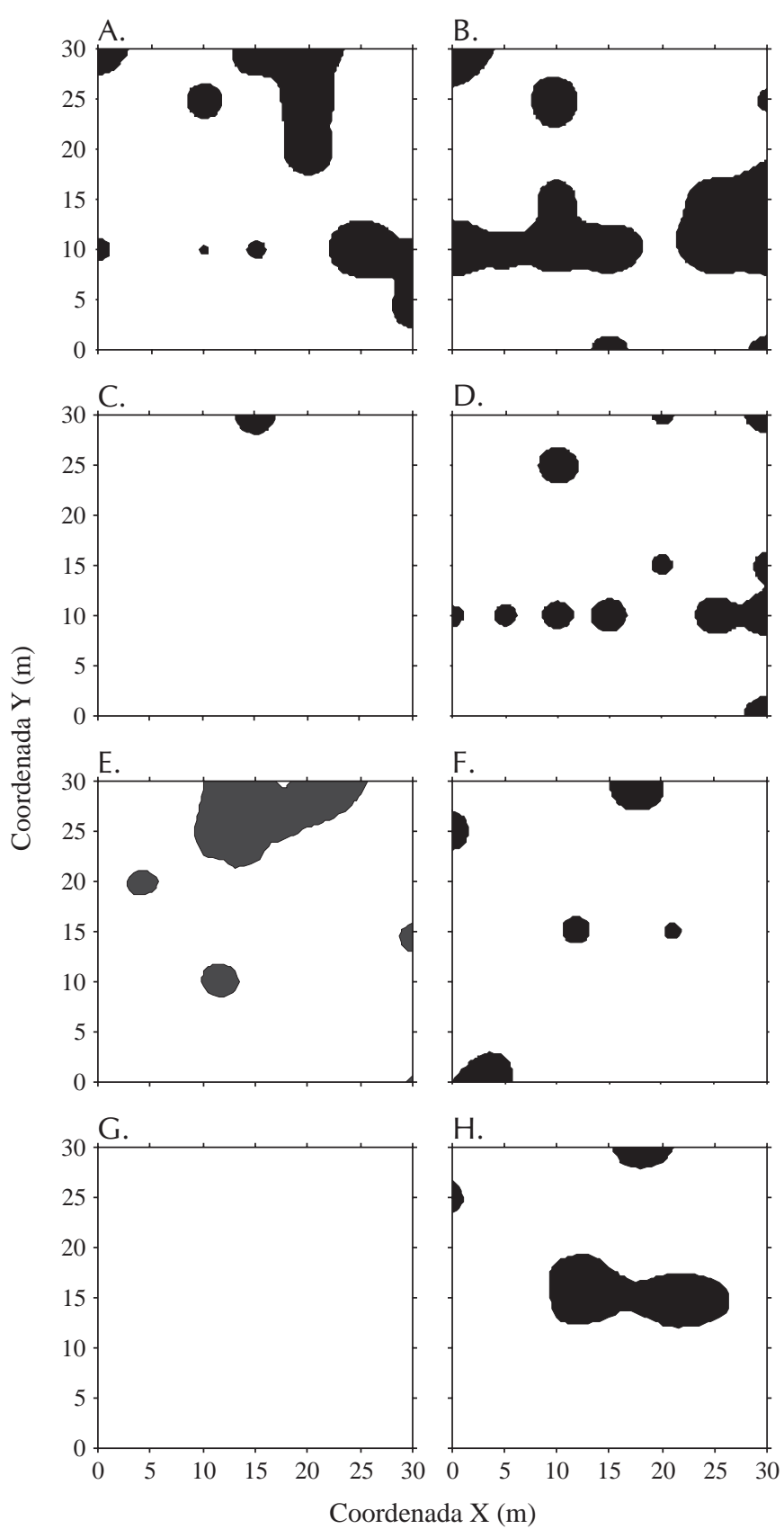

Figura 1. Mapas de isolinhas para $C E_{\text {s }}$, cujas áreas escuras representam condutividade elétrica $\geq 4,0 \mathrm{dS} \mathrm{m}^{-1}$. A: $\mathrm{S}_{1}(0-20 \mathrm{~cm}) 1^{\text {a }}$ coleta; $B: S_{1}(0-20 \mathrm{~cm})$ $2^{\text {a }}$ coleta; $C: S_{1}(20-40 \mathrm{~cm}) 1^{\text {a }}$ coleta; $D: S_{1}(20-40 \mathrm{~cm}) 2^{\text {a }}$ coleta; $E: S_{2}(0-20 \mathrm{~cm})$ $1^{\text {a }}$ coleta; $F: S_{2}(0-20 \mathrm{~cm}) 2^{\text {a }}$ coleta; $G: S_{2}(20-40 \mathrm{~cm}) 1^{\text {a }}$ coleta; $H: S_{2}(20-40 \mathrm{~cm})$ $2^{\mathrm{a}}$ coleta. A $1^{\mathrm{a}}$ coleta se refere ao cenário antes do experimento enquanto a $2^{\mathrm{a}}$ ocorreu aos 96 DAS centagens de 4,09 e 10,55\% (Figuras $1 \mathrm{~F}$ e $1 \mathrm{H}$ ) respectivamente. Fica evidenciado, portanto, que mesmo se adotando uma fração de lixiviação de 0,20 e água com salinidade média de 1,42 dS $\mathrm{m}^{-1}$, não foi possível evitar o acúmulo de sais da área inicialmente salina, exceto para o $\mathrm{S}_{2}(0-20 \mathrm{~cm})$. Ao se considerar a profundidade explorada pelas raízes, ou seja, a camada de 0-40 cm, observou-se, pela média das percentagens das áreas salinas, nas camadas $(0-20 \mathrm{~cm})$ e (20-40 cm), que no início do experimento ( $1^{\mathrm{a}}$ coleta) o $\mathrm{S}_{1}$ apresentava 7,98\% da área salinizada, aumentando no final do ciclo ( $2^{\mathrm{a}}$ coleta) para 15,09\% da área total; já para o $S_{2}$ houve um leve decréscimo, passando de 5,97\% ( $1^{\text {a }}$ coleta) para 5,52\% ( $2^{\text {a }}$ coleta). Esta redução está associada ao efeito combinado da textura e cobertura morta.

Nas Figuras 2A e 2B se encontra a distribuição de freqüência acumulada para a umidade do solo, para as camadas de 20 e $40 \mathrm{~cm}$, verificando-se que o $\mathrm{S}_{2}$ apresentou umidades maiores que o $S_{1}$, demonstrando o efeito positivo da cobertura morta na manutenção de umidade e, conseqüentemente, nas menores percentagens de áreas salinas; já as Figuras 2C e 2D ilustram a distribuição de freqüência acumulada para os teores de argila + silte, em que o $S_{1}$ apresenta valores muito superiores ao $S_{2}$, o que indica maior infiltração de água no $\mathrm{S}_{2}$. Resultado explicado pela diferença da textura nos setores. Com relação a PST, não se verificaram áreas sodificadas uma vez que não foram encontrados valores de PST iguais ou maiores de $15 \%$, para ambos os setores e camadas, nas épocas amostradas.

Com relação à produtividade total, o $S_{1}$ apresentou $43.494 \mathrm{~kg} \mathrm{ha}^{-1}$, enquanto para o $\mathrm{S}_{2}$ o valor foi de $36.309 \mathrm{~kg} \mathrm{ha}^{-1}$, avaliados a partir de 49 e 52 amostras, para os setores 1 e 2 , respectivamente. $\mathrm{O}$ menor valor de produtividade do $\mathrm{S}_{2}$, quando comparado com o $S_{1}$, pode ter sido devido aos maiores valores de umidade no $S_{2}$, resultantes da adição de cobertura morta. Os valores médios de produtividade obtidos foram superiores aos encontrados na região, que se situam entre 25.000 e $30.000 \mathrm{~kg} \mathrm{ha}^{-1}$; assim, se constata que, com um manejo eficiente da irrigação, baseado no fornecimento adequado de água e nutrientes, é possível alcançar produtividades satisfatórias mesmo quando a água de irrigação possui concentração moderada de sais. Resende \& Cordeiro (2007), trabalhando com três níveis de salinidade de água: $0,1 \mathrm{dS} \mathrm{m}^{-1}, 4,0 \mathrm{dS} \mathrm{m}^{-1}$ e $8,0 \mathrm{dS} \mathrm{m}^{-1}$ em solo não salino no cultivo de cenoura Brasília nas condições de Petrolina, PE, encontraram produtividades comerciais para os respectivos níveis de salinidade de 82.300, 61.000 e $33.100 \mathrm{~kg} \mathrm{ha}^{-1}$ em condições de solo não salino, demonstrando que é possível produzir cenoura no semi-árido brasileiro com as águas disponíveis, sendo necessário observar ainda a condição do solo após um cultivo, adotando-se águas com alta salinidade para evitar problemas de salinização e provável sodificação dos solos.

Os resultados apresentados neste trabalho sugerem a necessidade de estudos regionais com o propósito de revisar os limites de tolerância da salinidade para a cultura da cenoura estabelecidos por Ayers \& Westcot (1991), tendo em vista que se encontraram produtividades superiores às da média nacional de 2005, que correspondeu a $29.453 \mathrm{~kg} \mathrm{ha}^{-1}$ (FAOFAOSTAT, 2006) 


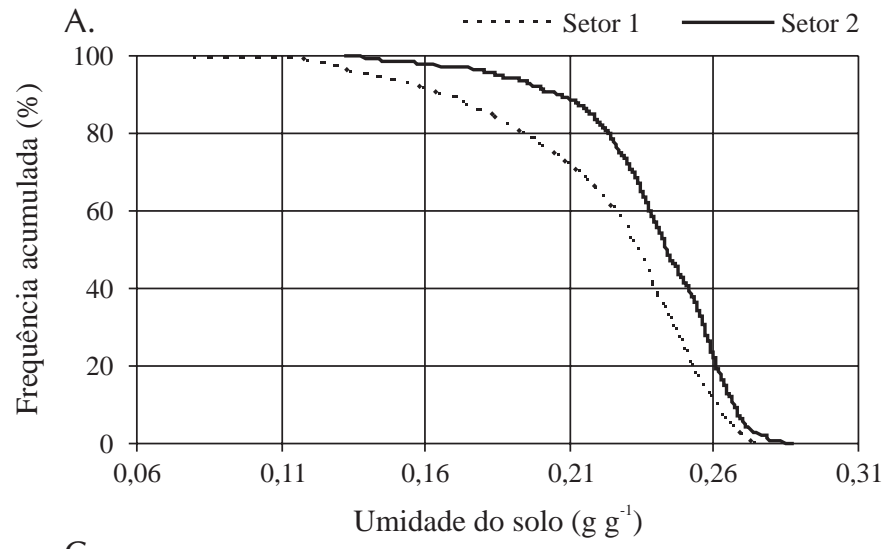

C.

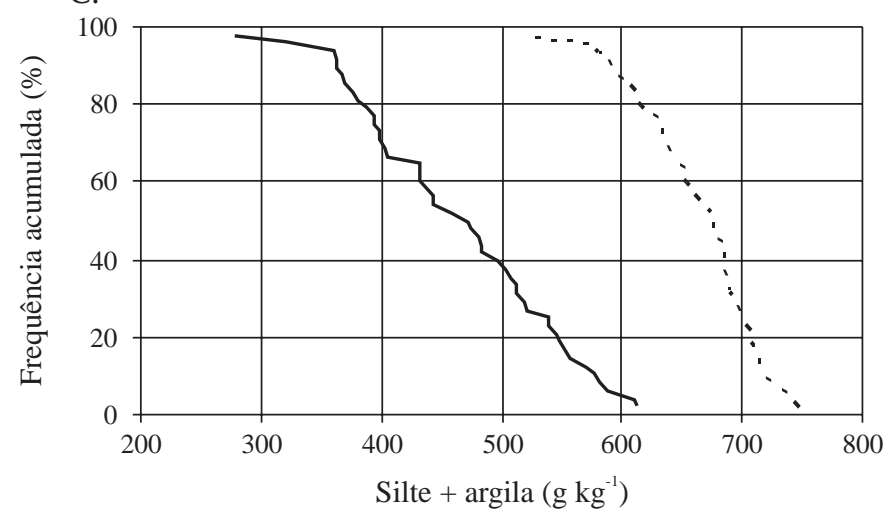

B.

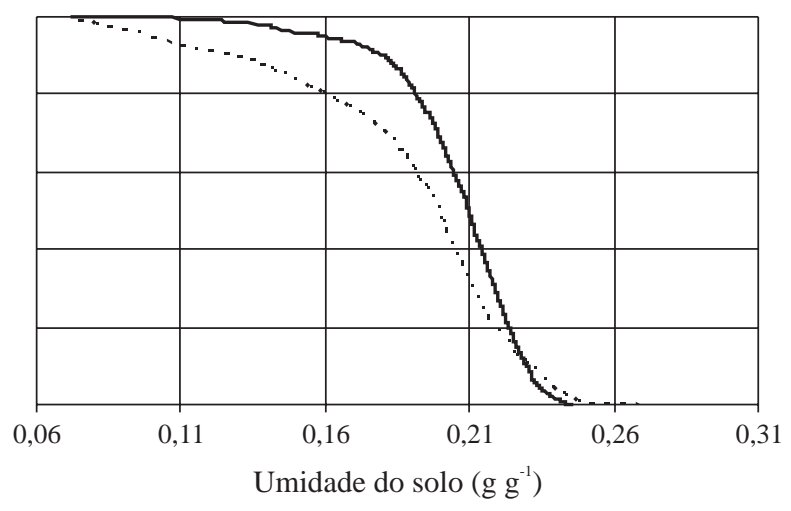

D.

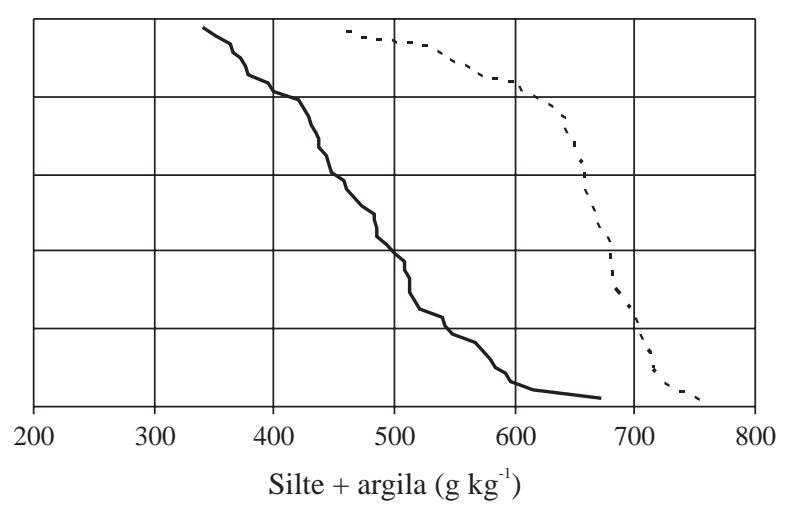

Figura 2. Distribuições de freqüência acumulada da umidade gravimétrica do solo a $20 \mathrm{~cm}(\mathrm{~A})$ e $40 \mathrm{~cm}(\mathrm{~B})$ e dos teores de argila + silte, $0-20 \mathrm{~cm}(\mathrm{C})$ e 20-40 cm (D) para os dois setores

\section{CONCLUSÕES}

1. Notou-se incremento dos valores médios da relação de adsorção de sódio durante o cultivo, nas camadas de 0-20 e 20-40 cm, nos dois setores.

2. Os valores de condutividade elétrica do extrato de saturação, a relação de adsorção de sódio e percentagem de sódio trocável, foram mais elevados na camada de 0-20 cm, para os dois setores, nas duas épocas de amostragem.

3. A fração de lixiviação aplicada possibilitou a manutenção da $\mathrm{CE}_{\mathrm{es}}$ média do solo na zona radicular, ao final do ciclo, uma vez que não houve diferença estatística quando se comparou com a média da salinidade inicial, exceto para o $\mathrm{S}_{1}(20-40 \mathrm{~cm})$.

4. Com o mapeamento da $\mathrm{CE}_{\mathrm{es}}$ e se considerando a camada de 0-40 cm, concluiu-se que a área salinizada no $\mathrm{S}_{1}$, inicialmente de 7,98\%, passou para 15,09\% após o ciclo da cultura. Já para o $\mathrm{S}_{2}$, com cobertura morta, a área salina passou de 5,97 para 5,52\%. Indicativo da influência da textura do solo e da cobertura morta.

5. A variável que apresentou maiores correlações no espaço amostral foi a PST, enquanto a $\mathrm{CE}_{\mathrm{es}}$ e a RAS apresentaram, em sua maioria, efeito pepita puro.

\section{AGRADECIMENTOS}

Os autores agradecem o apoio do CT-Hidro/CNPq, pelo financiamento do projeto e concessão das bolsas de mestra- do e doutorado do primeiro autor, bem como pelas significativas contribuições dos consultores.

\section{LITERATURA CITADA}

Audry, P.; Suassuna, J. A salinidade das águas disponíveis para pequena irrigação no sertão nordestino: Caracterização, variação sazonal, limitação de uso. Recife: Conselho Nacional de Desenvolvimento Científico e Tecnológico, 1995. 128p.

Ayers, R. S.; Westcot, D. W. A qualidade da água na agricultura. Campina Grande: UFPB, 1991. 218p. FAO. Estudos de Irrigação e Drenagem, 29.

Blanco, F. F.; Folegatti, M. V. Salt accumulation and distribution in a greenhouse soil as affected by salinity of irrigation water and leaching management. Revista Brasileira de Engenharia Agrícola e Ambiental, v.6, n.3, p.414-419, 2002.

Cambardella, C. A.; Moorman, T. B.; Novak, J. M.; Parkin, T. B.; Karlen, D. L.; Turco, R. F.; Kornopka, A. E. Field-scale variability of soils properties in central Iowa Soils. Soil Science Society of American Journal, v.58, p.1501-1511. 1994.

CEFS - Comissão Estadual de Fertilidade do Solo. Recomendações de adubação para o Estado de Pernambuco. (2 $2^{\mathrm{a}}$ aproximação) 2.ed.. Recife: Instituto de Pesquisa Agropecuária, 1998. 198p

Dias, N. da S.; Duarte, S. N.; Gheyi, H. R.; Medeiros, J. F. de; Soares, T. M. Manejo da fertirrigação e controle da salinidade do solo sob ambiente protegido, utilizando-se extratores de solução do solo. Revista Brasileira de Engenharia Agrícola e Ambiental, v.9, n.4, p.496-504, 2005. 
Dias, N. da S.; Medeiros, J. F. de; Gheyi, H. R.; Silva, F. V.; Barros, A. D. de. Evolução da salinidade em um Argissolo sob cultivo de melão irrigado por gotejamento. Revista Brasileira de Engenharia Agrícola e Ambiental, v.8, n.2/3, p.240-246, 2004.

Doorenbos, J.; Pruitt, W. O. Guidelines for predicting crop water requirements. Rome, FAO, 1977. 179p. FAO Irrigation and Drainage, Paper n. 24.

EMBRAPA - Empresa Brasileira de Pesquisa Agropecuária. Manual de métodos de análise de solo. Rio de Janeiro: EMBRAPA. 1997. 212p.

FAO-FAOSTAT. Food and Agriculture Organization of the United Nations. Produtividade da cenoura:<http://apps.fao.org $>18$ Dez. 2006

Freire, M. B. G. S. Saturação por sódio e qualidade da água de irrigação na degradação de propriedades físicas de solos do Estado de Pernambuco. Viçosa: UFV, 2001. 66p. Tese Doutorado

Freire, M. B. G. S.; Ruiz, H. A.; Ribeiro, M. R.; Ferreira, P. A.; Alvarez, V. H.; Freire, F. J. Condutividade hidráulica de solos de Pernambuco em resposta à condutividade elétrica e RAS da água de irrigação. Revista Brasileira de Engenharia Agrícola e Ambiental, v.7, n.1, p.45-52, 2003a.

Freire, M. B. G. S.; Ruiz, H. A.; Ribeiro, M. R.; Ferreira, P. A.; Alvarez, V. H.; Freire, F. J. Estimativa do risco de sodificação de solos de Pernambuco pelo uso de águas salinas. Revista Brasileira de Engenharia Agrícola e Ambiental, v.7, n.2, p.227-232, 2003b.

Horney, R. D.; Taylor, B.; Munk, D. S.; Roberts, B. A.; Lesch, M.; Plant, R. E. Development of practical site-specific management methods for reclaiming salt-affected soil. Computers and Electronics in Agriculture, v.46, p.379-397, 2005.

Resende, G. M.; Cordeiro, G. G. Produtividade da cenoura em função da qualidade da água e condicionador de solo no Vale do São Francisco. Caatinga, v.20, n.1, p.100-104, 2007.
Ribeiro, M. R.; Corrêa, M. M. Levantamento detalhado de solos da Fazenda Nossa Senhora do Rosário. In: Montenegro, A. A. A. Diagnóstico e controle da salinização em aluviões com pequena agricultura irrigada. Recife: UFRPE, 2001. Relatório de Projeto CNPq Programa Nordeste

Ribeiro, M. R; Freire, F. J; Montenegro, A. A. A. Solos halomórficos no Brasil: Ocorrência, gênese, classificação, uso e manejo sustentável. In: Tópicos em Ciência do Solo. Viçosa: SBCS, v.3, 2003. 430p.

Rhoades, J. D. Drainage for salinity control. In: van Schilfgaarde J. (ed.). Drainage for agriculture. Madison: American Society of Agronomy, 1974. p.443-62. Monograph, 17

Richards, L. A. Diagnosis and improvement of saline and alkali soils. Washington: United States Salinity Laboratory. 1954. 160p.USDA: Agriculture Handbook, 60.

Robertson, G. P. GS+: Geostatistics for the environmental sciences - GS+ User's Guide. Plainwell: Gamma Design Soffware, 1998. 152p.

Shapiro, S. S.; Wilk, M. B. An analysis of variance test for normality (complete sample). Biometrika, v.52, n.3/4, p.591-611, 1965.

Surfer. Surfer 8.0: Contouring and 3d surface mapping for scientists and engineers. User's Guide. New York: Golden Software, 2002. 619p.

Thomas, G. W. Exchangeable cations. In: Page, A. L. (ed). Methods of soil analysis. Part-2 chemical methods. Madison: American Society of Agronomy, 1982, p.159-165.

Vauclin, M.; Vieira, S. R.; Vauchaud, G.; Nielsen, D. R. The use of cokriging with limited field soil observations. Soil Science Society of America Journal, v.47, n.1, p.175-184, 1983.

Vieira, S. R.; Millete, J.; Topp, G. C.; Reynolds, W. D. Handbook for geoestatistical analysis of variability in soil and climate data. In: Tópicos em ciência do solo, v.II, Viçosa: SBCS, 2002. 692p.

Warrick, A. W.; Nielsen, D. R. Spatial variability of soil physical properties in the field. In: Hillel, D. (ed.). Applications of soil physics. New York: Academic Press, 1980. Cap.2, p.319-344. 